\title{
Chemische Brennstoffe aus Sonnenlicht
}

\section{Report}

Author(s):

Meier, Anton; Palumbo, Robert; Steinfeld, Aldo (D)

Publication date:

2001

Permanent link:

https://doi.org/10.3929/ethz-a-004486058

\section{Rights / license:}

In Copyright - Non-Commercial Use Permitted 


\title{
CHEMISCHE BRENNSTOFFE AUS SONNENLICHT
}

\author{
Anton Meier ${ }^{1}$, Robert Palumbo ${ }^{1,2}$ und Aldo Steinfeld ${ }^{1,3}$ \\ ${ }^{1}$ Labor für Solartechnik, Forschungsbereich Allgemeine Energie \\ Paul Scherrer Institut, CH-5232 Villigen PSI, Schweiz \\ E-mail: anton.meier@psi.ch \\ ${ }^{2}$ Department of Mechanical Engineering \\ Valparaiso University, Indiana, USA \\ E-mail: robert.palumbo@psi.ch \\ ${ }^{3}$ Institut für Energietechnik, Abteilung für Maschinenbau und Verfahrenstechnik \\ ETH - Eidgenössische Technische Hochschule, CH-8092 Zürich, Schweiz \\ E-mail: aldo.steinfeld@eth.ch
}

\section{ZUSAMMENFASSUNG}

Sonnenenergie kann in speicherbare und transportierbare chemische Brennstoffe wie Wasserstoff und Methanol umgewandelt werden. Um die benötigte Prozesswärme bei Temperaturen von über $2300 \mathrm{~K}$ zu erzeugen, wird das Sonnenlicht mittels Parabolspiegeln konzentriert. Solare Hochtemperaturwärme wird bei der Herstellung von Metallen und Zement sowie bei der Wiederverwertung von schwermetallhaltigen Abfallstoffen eingesetzt. Dadurch lässt sich der Ausstoss von Treibhausgasen und anderen Schadstoffen vermeiden, die bei der Verbrennung von fossilen Brennstoffen für die Wärme- und Stromproduktion anfallen.

Die Forschung auf dem Gebiet der Hochtemperatur-Solarchemie wird in der Schweiz am Paul Scherrer Institut (PSI) in Villigen und an der Eidgenössischen Technischen Hochschule (ETH) in Zürich betrieben und umfasst sowohl grundlegende Studien chemischer Reaktionen als auch die Entwicklung solarchemischer Reaktoren.

\section{EINLEITUNG}

Stellen Sie sich vor, Sie wären an einem trüben, regnerischen Novemberabend mit Ihrem Auto unterwegs, irgendwo im Norden Europas. Sie können kaum die Strasse vor ihnen erkennen. Sie bemerken, dass der Benzintank fast leer ist. Sie fahren zur nächsten Tankstelle und betanken Ihr Auto mit Sonnenlicht und Wasser. Sonnenlicht und Wasser? Ja, tatsächlich. Sonnenlicht und Wasser sind auf der Erdoberfläche reichlich vorhanden. Und dennoch denken wir normalerweise nicht daran, diese natürlichen Reichtümer zu nutzen, um allfällige künftige Energiekrisen zu bewältigen, geschweige denn, um daraus Brennstoffe zu entwickeln, die unseren Städten saubere Luft bescheren würden. Wahrscheinlich fehlt uns einfach die Vorstellungskraft, um zu erkennen, dass mit Hilfe dieser Ressourcen die dringendsten Probleme des angehenden 21. Jahrhunderts gelöst werden könnten: die drohende Verknappung der Erdölreserven und die zunehmende Umweltverschmutzung.

Gleichwohl sind WissenschaftlerInnen und IngenieurInnen rund um die Welt fasziniert von einer ziemlich verblüffenden Tatsache: Auf lediglich $0.1 \%$ des verfügbaren Landes liesse sich mittels Solaranlagen mit einem Wirkungsgrad von 20\% soviel Energie gewinnen, dass der gegenwärtige Energiebedarf sämtlicher Bewohner dieses Planeten gedeckt werden könnte. Die Sonnenenergie ist im wesentlichen uneingeschränkt vorhanden und ihre Verwendung ist 
ökologisch sinnvoll. Genügend gute Gründe also für eine ausgedehnte Nutzung der Sonnenenergie, wenn da nicht ein paar ernsthafte Nachteile wären: die auf die Erde treffende Solarstrahlung ist stark verdünnt (nur etwa $1 \mathrm{~kW} / \mathrm{m}^{2}$ ), nicht dauernd verfügbar (nur am Tag und bei klarem Himmel) sowie ungleichmässig über die Erdoberfläche verteilt (vorwiegend in Wüstenregionen zwischen $30^{\circ}$ nördlicher und $30^{\circ}$ südlicher Breite, wie aus Abbildung 1 hervorgeht).

Die auf dem Gebiet der Sonnenenergie tätigen ForscherInnen sind überzeugt, dass diese Nachteile überwindbar sind. Sie suchen nach Rezepten, um die Sonnenenergie einzufangen, damit sie konzentriert, gespeichert und schliesslich von den sonnigen und spärlich besiedelten Gebieten im Sonnengürtel der Erde zu den industrialisierten und stark bevölkerten Zentren transportiert werden kann, wo die meiste Energie benötigt wird. Sie schlagen vor, konzentrierte Solarenergie in chemische Energieträger umzuwandeln, und zwar in Form von solaren Brennstoffen, die über lange Zeit gespeichert und über weite Distanzen transportiert werden können. Autos, die mit solchen Brennstoffen angetrieben werden, fahren in der Tat mit Sonnenenergie. Diese WissenschaftlerInnen sehen die Sonnenenergie buchstäblich in Bewegung.

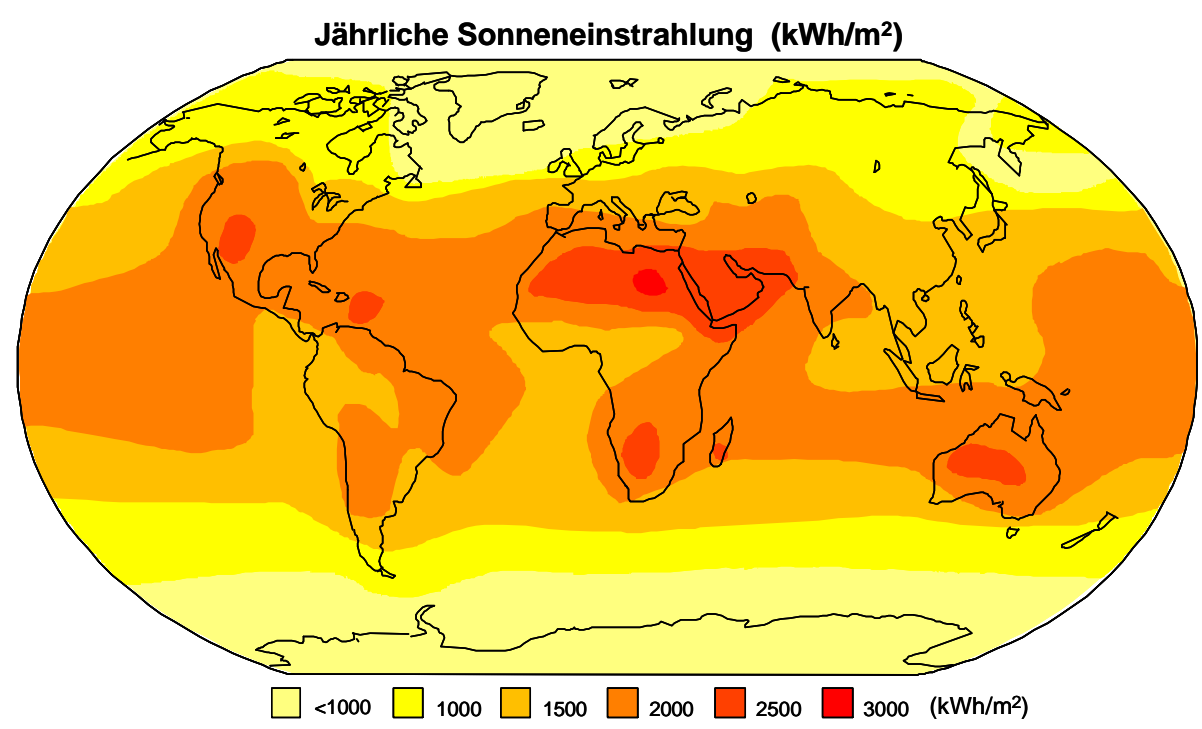

\begin{abstract}
Abbildung 1: Weltweite Verteilung der jährlichen Sonneneinstrahlung. Die Gebiete mit der grössten Sonneneinstrahlung liegen zwischen $30^{\circ}$ nördlicher und $30^{\circ}$ südlicher Breite. Nimmt man eine mittlere jährliche Einstrahlung von 2350 $\mathrm{kWh} / \mathrm{m}^{2}$ und einen solaren Umwandlungswirkungsgrad von nur $20 \%$ an, genügt es, eine Fläche von etwa $500 \mathrm{~km}$ x $500 \mathrm{~km}$ mit Sonnenkollektoren zu bestücken, um den gesamten jährlichen Weltenergiebedarf $\left(1,1 \times 10^{14} \mathrm{kWh}\right) \mathrm{zu}$ decken [1].
\end{abstract}

\title{
THERMOCHEMISCHE UMWANDLUNG DER SONNENENERGIE
}

\section{Thermodynamisches Prinzip}

Die Werkzeuge, mit denen aus Sonnenlicht Brennstoffe für das 21. Jahrhundert hergestellt werden können, finden sich schon in den Schriften zweier herausragender Gelehrter des 19. Jahrhunderts, Carnot und Gibbs. Die beiden Wissenschaftler gehörten zu den wichtigsten Begründern der Thermodynamik. Das ist die Lehre, welche beschreibt, wie Energie von einer Form in eine andere verwandelt werden kann, so z. B. solare Energie in chemische Energie. Etwas vereinfacht ausgedrückt, besagt die Thermodynamik: je höher die Temperatur der Sonnenenergie ist, die wir unserem Prozess zuführen, desto mehr können wir aus dem 
entstandenen Endprodukt machen. Betrachten wir zum Beispiel einen typischen WarmwasserSonnenkollektor, mit dem wir Wasser zum Duschen oder für die Raumheizung erwärmen können. Obwohl eine solche Vorrichtung für lokale Anwendungen durchaus Sinn machen kann, lässt sich beispielsweise die auf diese Art in Australien gesammelte Sonnenenergie nicht nach Tokio transportieren. Wird aber Sonnenenergie bei sehr hohen Temperaturen (nahezu $2300 \mathrm{~K})$ einem chemischen Reaktor zugeführt, eröffnen sich interessante Perspektiven: Sonnenenergie, die in Australien gespeichert wurde, kann Häuser heizen, Strom liefern, Autos antreiben, und mehr... in Tokio!

\section{Konzentration des Sonnenlichts}

In Abbildung 2 ist dargestellt, wie die chemische Umwandlung von Sonnenenergie funktioniert: wenn wir das einfallende Sonnenlicht mit Hilfe eines parabolischen Spiegels auf eine kleine Fläche konzentrieren und dann diese Strahlungsenergie mit einem geeigneten Empfänger einfangen, können wir mit der auf diese Weise erzeugten Hochtemperaturwärme chemische Umwandlungen durchführen und dadurch speicherbare und transportierbare Brennstoffe produzieren. Unabhängig von der Art dieses Brennstoffs ist der maximale Wirkungsgrad eines solchen Energieumwandlungsprozesses durch den Carnot-Wirkungsgrad einer äquivalenten Wärmekraftmaschine begrenzt: ist die Sonnenoberfläche ein Wärmereservoir bei $5800 \mathrm{~K}$ und die Erde eine Wärmesenke, könnten im Prinzip 95\% der Sonnenenergie in chemische Energie von Brennstoffen umgewandelt werden. Natürlich liegt es an uns, eine geeignete Technologie zu entwerfen und zu entwickeln, um möglichst nahe an diese Grenze heranzukommen.

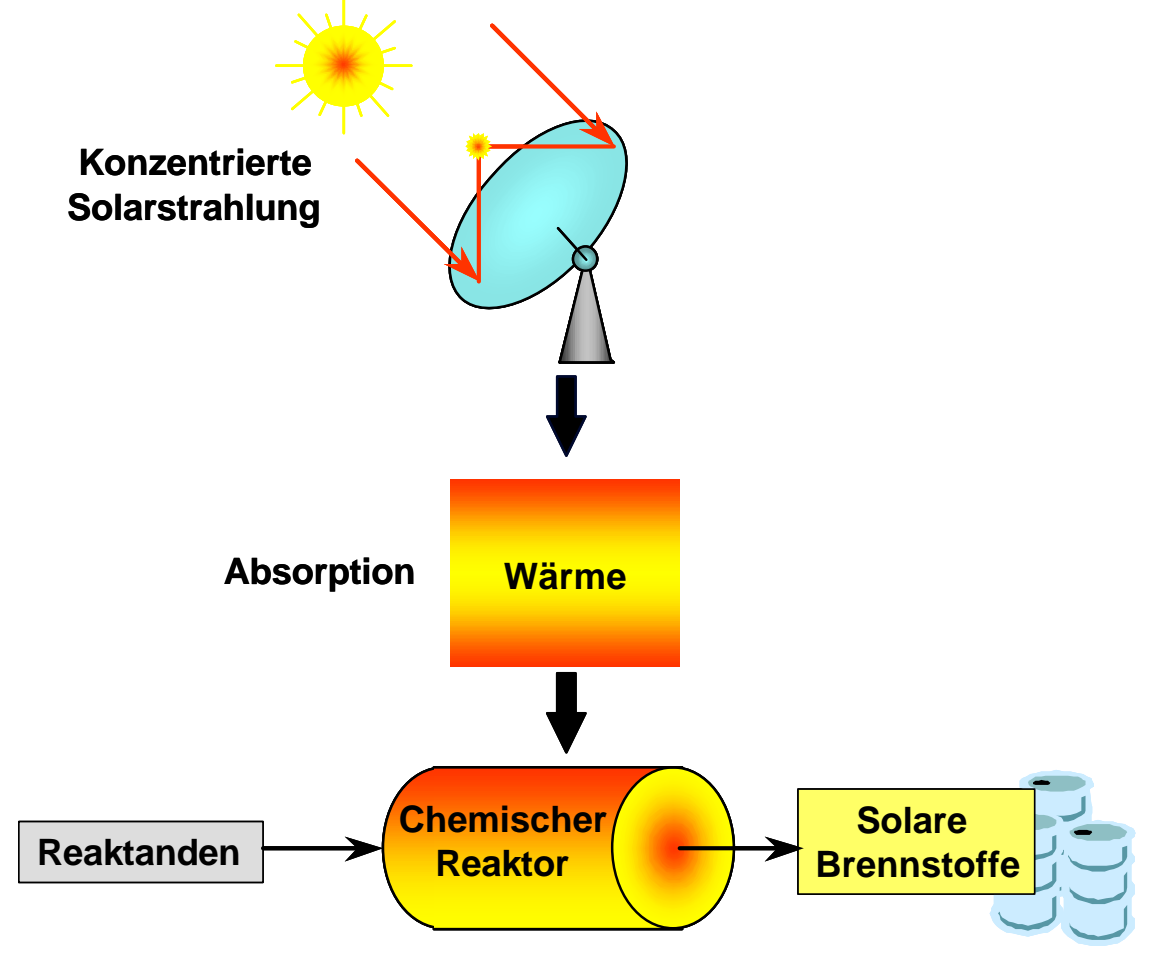

Abbildung 2: Schematische Darstellung der Umwandlung von Sonnenenergie in solare Brennstoffe. Konzentrierte Solarstrahlung dient als Energiequelle für die Hochtemperatur-Prozesswärme, womit chemische Reaktionen für die Herstellung von speicherbaren und transportierbaren Brennstoffen angetrieben werden [2]. 
Wir alle wissen aus eigener Erfahrung, dass an heissen Sommertagen Beton und schwarze Oberflächen warm werden. Einige von uns können sich sogar noch daran erinnern, wie man mit einem Vergrösserungsglas Blätter und Zweige entzünden kann, doch es ist wahrscheinlich für die meisten Leute schwierig, sich vorzustellen, dass Sonnenlicht in weniger als 10 Sekunden fussballgrosse Löcher durch zentimeterdicke Stahlplatten bohren kann. Aber dies ist möglich. Das Prinzip ist dasselbe wie beim Vergrösserungsglas, doch statt mit einer Linse wird das Licht mit einem Parabolspiegel fokussiert. Abbildung 3 zeigt einen solchen Solarkonzentrator am Paul Scherrer Institut (PSI). Dieser Parabolspiegel folgt der Sonne auf ihrer Bahn am Himmel und reflektiert die Sonnenstrahlen im wesentlichen auf eine kleine Kreisfläche. Die Energie, welche auf diese kleine Fläche auftrifft, ist gleichbedeutend mit derjenigen von 5000 Sonnen. Mit dieser Technologie können wir leicht jene sehr hohen Temperaturen erreichen, die für die Produktion von solaren Brennstoffen benötigt werden.

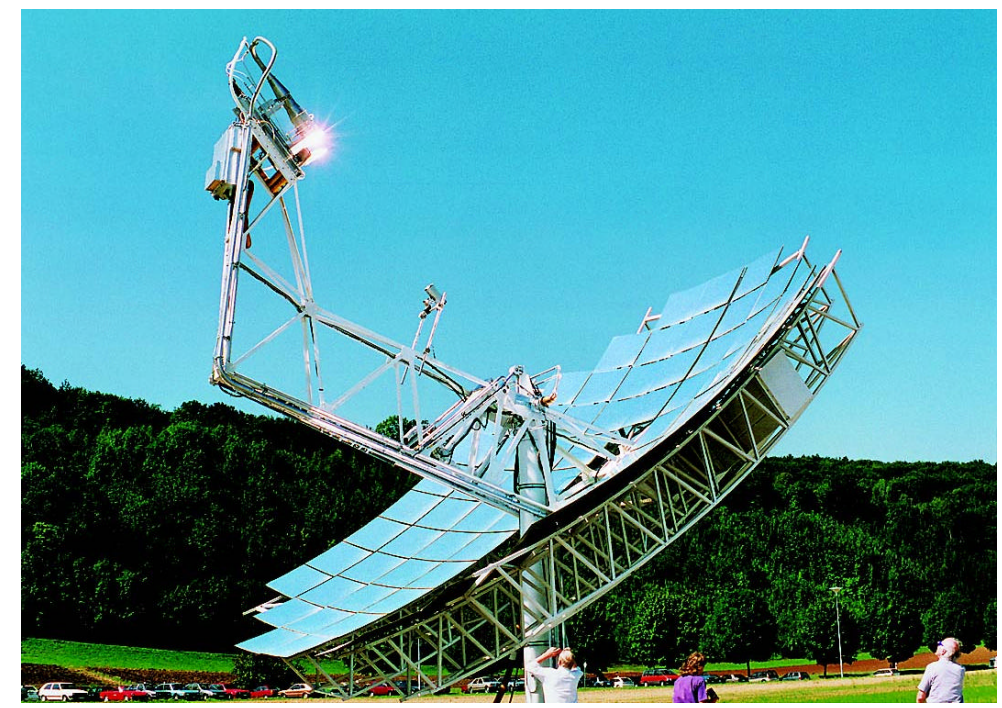

Abbildung 3: Der Parabolspiegel am Paul Scherrer Institut (PSI) folgt der Sonnenbahn und konzentriert die Sonnenstrahlen 5000-fach auf einen kleinen Kreis in der Brennebene.

\section{THERMOCHEMISCHE PROZESSE MIT SONNENENERGIE}

\section{Solarthermische Produktion von Wasserstoff}

Ein potentiell sehr attraktiver chemischer Prozess ist die solarthermische Produktion von Wasserstoff aus Wasser. Bei hohen Temperaturen über $2500 \mathrm{~K}$ und abhängig vom Druck spaltet sich Wasser in Wasserstoff und Sauerstoff. Dieser Prozess wird als Thermolyse von Wasser bezeichnet:

$$
\mathrm{H}_{2} \mathrm{O} \rightarrow \mathrm{H}_{2}+\frac{1}{2} \mathrm{O}_{2}
$$

Die direkte Wasserspaltung wäre ein genial einfaches Konzept, doch fehlt bislang eine wirksame Technik zur Trennung des Wasserstoffs vom Sauerstoff bei hohen Temperaturen, ohne dass es zu einer Explosion kommt. Dieses Trennungsproblem kann umgangen werden mittels thermochemischer Wasserspaltungs-Zyklen, bei denen Wasserstoff und Sauerstoff in verschiedenen Schritten anfallen. Als Beispiel betrachten wir zweistufige WasserspaltungsZyklen, die auf sogenannten Metalloxid-Redox-Systemen basieren. Der erste, solare, endotherme Schritt umfasst die solarthermische Dissoziation von Metalloxiden bei hohen Temperaturen: 


$$
\mathrm{M}_{x} \mathrm{O}_{y} \rightarrow x M+y / 2 \mathrm{O}_{2}
$$

Der zweite, nicht-solare, exotherme Schritt ist die Hydrolyse der entstandenen Metalle bei moderaten Temperaturen (unter etwa $900 \mathrm{~K}$ ), wobei molekularer Wasserstoff und die entsprechenden Metalloxide gebildet werden:

$$
x \mathrm{M}+\mathrm{yH}_{2} \mathrm{O} \rightarrow \mathrm{M}_{x} \mathrm{O}_{y}+y \mathrm{H}_{2}
$$

Mit solchen Reduktions-Oxidations-Systemen lassen sich Wirkungsgrade über 30\% erzielen, wenn man die Solarstrahlung sehr stark konzentriert und die Wärme, die beim Abkühlen der Produkte anfällt, wieder zurückgewinnen kann [3].

Wie diese Idee funktioniert, zeigt Abbildung 4 schematisch am Beispiel des Zinkoxid-RedoxSystems. In einem ersten Schritt wird in einem sonnigen Gebiet der Erde Zinkoxid in den Brennpunkt eines Solarkonzentrators gebracht. Bei einer Temperatur von nahezu $2300 \mathrm{~K}$ zerfällt Zinkoxid in metallisches Zink (Reduktion). Der dabei frei werdende Sauerstoff kann entweder aufgefangen oder einfach an die Atmosphäre abgegeben werden. In einem zweiten Schritt, bei dem die Sonne nicht benötigt wird, lässt man Zink mit Wasser reagieren, wobei Wasserstoff frei wird. Dabei verwandelt sich Zink zurück in Zinkoxid (Oxidation), welches wieder dem solaren Schritt zugeführt werden kann. Der Wasserstoff kann entweder direkt zur Stromproduktion verwendet oder weiterverarbeitet werden, um andere Brennstoffe wie Methanol herzustellen. Nach der Energienutzung wird Wasserstoff wieder zu Wasser. Dies ist also ein geschlossener Kreislauf, bei dem kein Material verbraucht wird. Die einzige Energie, die dem Prozess zugeführt wird, ist das Sonnenlicht. Die Energie, die auf diese Art im Wasserstoff gespeichert wird, ist somit „,versteckte“ Sonnenenergie.

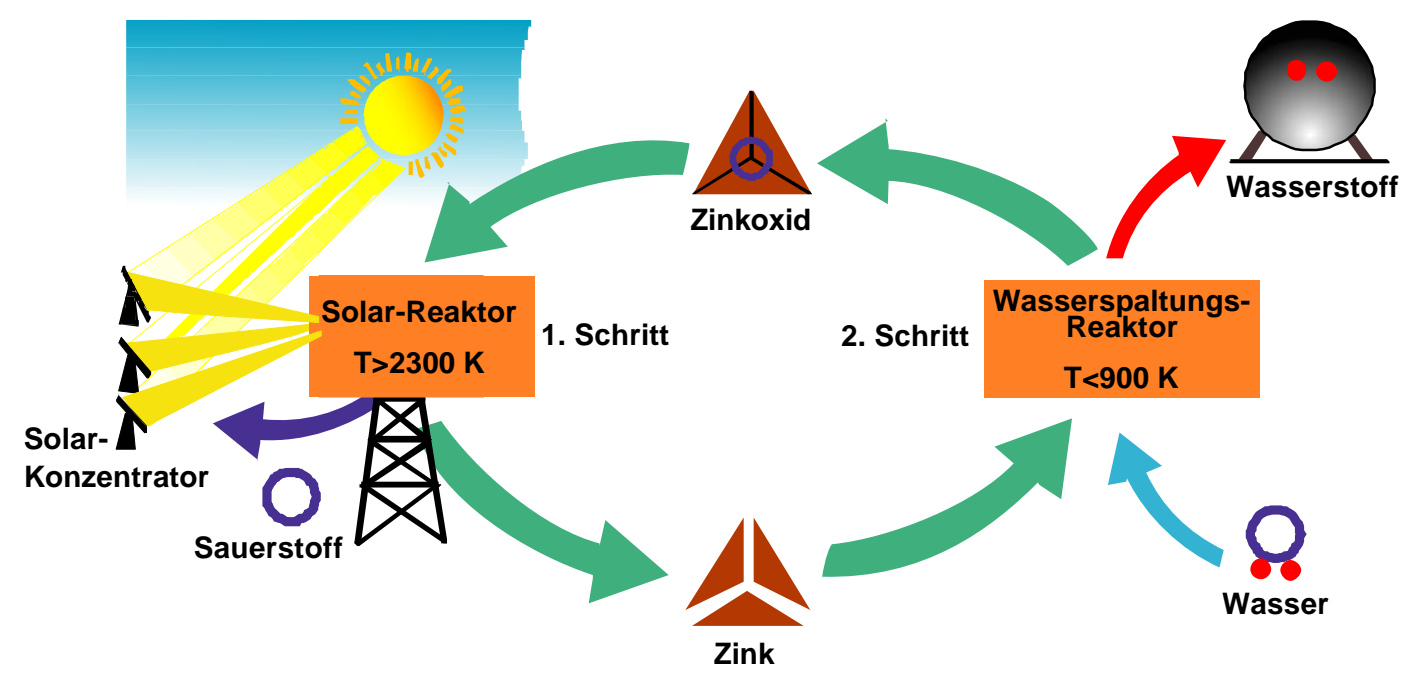

\begin{abstract}
Abbildung 4: Beispiel eines zwei-stufigen thermochemischen Kreissystems für die solare Herstellung von Wasserstoff aus Wasser. Im ersten, solaren Schritt wird Zinkoxid bei ca. $2300 \mathrm{~K}$ mit Hilfe von solarer Prozesswärme reduziert. Im zweiten, nicht-solaren Schritt reagiert das reduzierte Zink mit Wasser und produziert Wasserstoff. Das Zink wird dabei wieder in ZnO umgewandelt und in den solaren Schritt zurückgeführt. Die Netto-Reaktion ist in Gl. (1) angegeben; Wasserstoff und Sauerstoff werden in verschiedenen Schritten erhalten, so dass die schwierige Trennung der Gase bei hohen Temperaturen hinfällig wird.
\end{abstract}

\title{
Solarthermische Produktion von Zink
}

Metalle wie Zink sind attraktive Kandidaten für die Speicherung und den Transport von Solarenergie. Metallisches Zink wird heutzutage vorwiegend in der galvanischen und chemischen Industrie eingesetzt. Zink eignet sich ausserdem als kompakter, fester Brennstoff, 
dessen Handhabung an Luft problemlos ist und der in Zink-Luft Brennstoffzellen und Batterien Verwendung findet. Allerdings verursachen die gegenwärtigen industriellen Verfahren zur Herstellung von Zink (und anderen Metallen) schwerwiegende ökologische Probleme auf Grund des hohen Energieverbrauchs und des damit verbundenen Schadstoffausstosses. Die metallurgische Industrie belastet die Umwelt mit enormen Mengen an Treibhausgasen und anderen Schadstoffen, die hauptsächlich bei der Verbrennung fossiler Brennstoffe zur Wärmeund Stromerzeugung anfallen. Diese Emissionen könnten bedeutend reduziert oder sogar vollständig eliminiert werden, wenn die Hochtemperatur-Prozesswärme mit einer alternativen Energiequelle, beispielsweise mit konzentrierter Sonnenenergie, bereitgestellt würde.

Ohne Zugabe von Reduktionsmitteln werden Temperaturen von über $2000 \mathrm{~K}$ benötigt, um Zink durch direkte solarthermische Dissoziation von Zinkoxid zu erhalten:

$$
\mathrm{ZnO}(s) \rightarrow \mathrm{Zn}(g)+1 / 2 \mathrm{O}_{2}
$$

Die gasförmigen Produkte, $\mathrm{Zn}(\mathrm{g})$ und $\mathrm{O}_{2}$, müssen sehr rasch abgekühlt werden, um die Rückreaktion zu verhindern. Die Wirksamkeit dieser Abschreckung ist einerseits abhängig von der Verdünnung der Produktgase mit einem Inertgas, anderseits von der Temperatur der Oberfläche, auf der die Produkte abgeschreckt werden. Eine thermodynamische Analyse dieses Prozesses verspricht einen maximalen Exergie-Wirkungsgrad von 55\% [4].

Die Solarchemie-Forschung am PSI und an der ETH verbindet grundlegende physikalische und chemische Studien mit verfahrenstechnischen Untersuchungen. Das Verständnis der äusserst komplexen Wechselwirkungen zwischen der konzentrierten Solarstrahlung, dem eingebrachten Reaktionsmaterial und der chemischen Reaktionskinetik ist unabdingbar für die Entwicklung von geeigneten Reaktoren, in denen die Sonnenenergie effizient in chemische Brennstoffe umgewandelt werden kann. Ein derartiger solarchemischer Reaktor ist in Abbildung 5 schematisch dargestellt [5].

Diese Reaktorkonfiguration besteht aus einem rotierenden Hohlraum-Empfänger ("Receiver"), der mit einer dicken Schicht aus pulverförmigem $\mathrm{ZnO}$ ausgekleidet ist. Diese Zinkoxid-Schicht wird durch die Zentrifugalkraft gegen die Reaktorwand gedrückt. Das Zinkoxid ist direkt der hochkonzentrierten Solarstrahlung ausgesetzt, die durch ein Fenster in den Reaktorraum gelangt. Das Zinkoxid dient gleichzeitig als Strahlungsabsorber, Wärmeisolator und chemischer Reaktand. Erste Untersuchungen mit einem $10 \mathrm{~kW}$ Reaktorprototyp wurden im HochflussSolarofen des PSI durchgeführt (Abbildung 6), wobei der Reaktor einer maximalen solaren Strahlungsintensität von über $3900 \mathrm{~kW} / \mathrm{m}^{2}$ ausgesetzt wurde. Auf der Zinkoxid-Oberfläche wurden Temperaturen von über $2200 \mathrm{~K}$ erreicht. Bei einer Heizrate von über $1000 \mathrm{~K} / \mathrm{sec}$ verhielt sich das Reaktorsystem thermisch träge und war gegenüber plötzlichen Temperaturschwankungen unempfindlich. Eine indirekt beheizte Version dieses Solarreaktors wurde ebenfalls untersucht, wobei eine Graphitkavität durch die konzentrierte Solarstrahlung aufgeheizt wurde. In diesem Fall erfolgte die Wärmeübertragung auf die Zinkoxid-Schicht durch Wärmeleitung und Wärmestrahlung. Die weiteren Arbeiten bei der Reaktorentwicklung konzentrieren sich darauf, optimale Betriebsbedingungen zur Erreichung eines maximalen Exergie-Wirkungsgrads zu finden. 

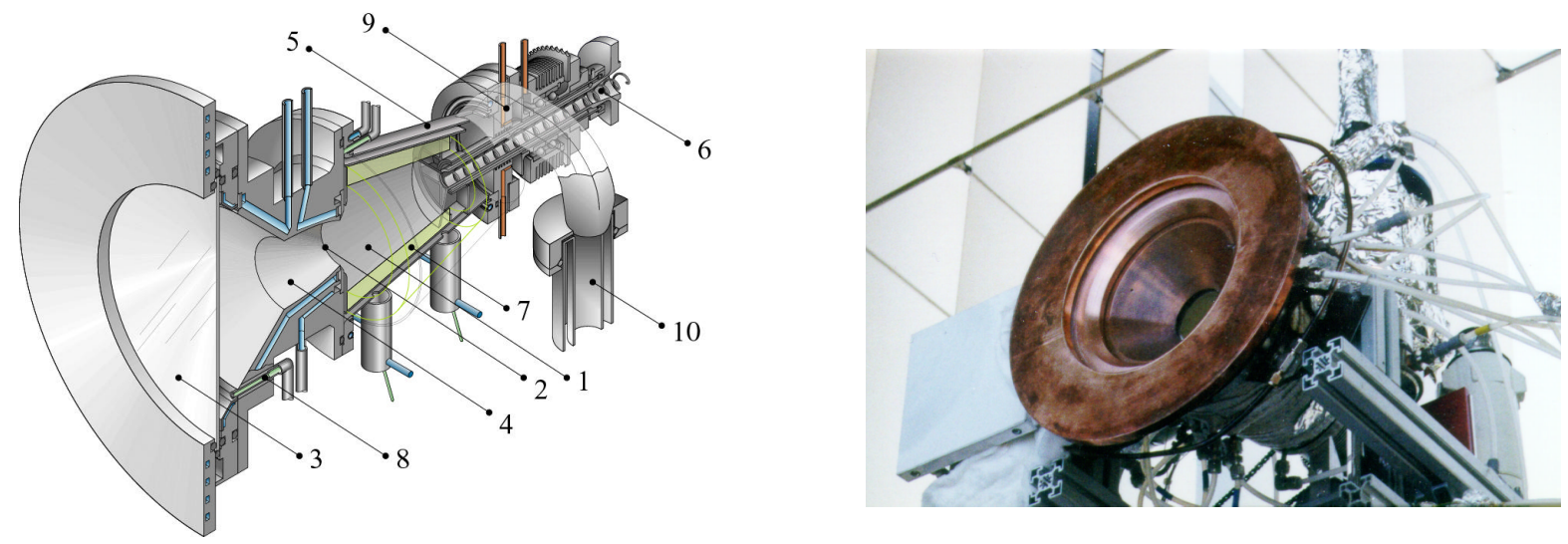

Abbildung 5: Schema (links) and Photo (rechts) eines solarchemischen Reaktors für die solarthermische Dissoziation von $\mathrm{ZnO}$, der am PSI entwickelt wird [5]. Legende: 1= rotierender Hohlraum-Empfänger ("Receiver"), 2= Eintrittsöffnung für konzentrierte Solarstrahlung, 3= Quarzfenster, 4= Sekundärkonzentrator $(\mathrm{CPC}=$ "Compound Parabolic Concentrator"), 5= äussere konische Wand, 6= ZnO Förderanlage, $7=\mathrm{ZnO}$ Schicht, 8= Einlass für Inertgase, 9= Auslass für Produktgase, 10= Abkühlvorrichtung.

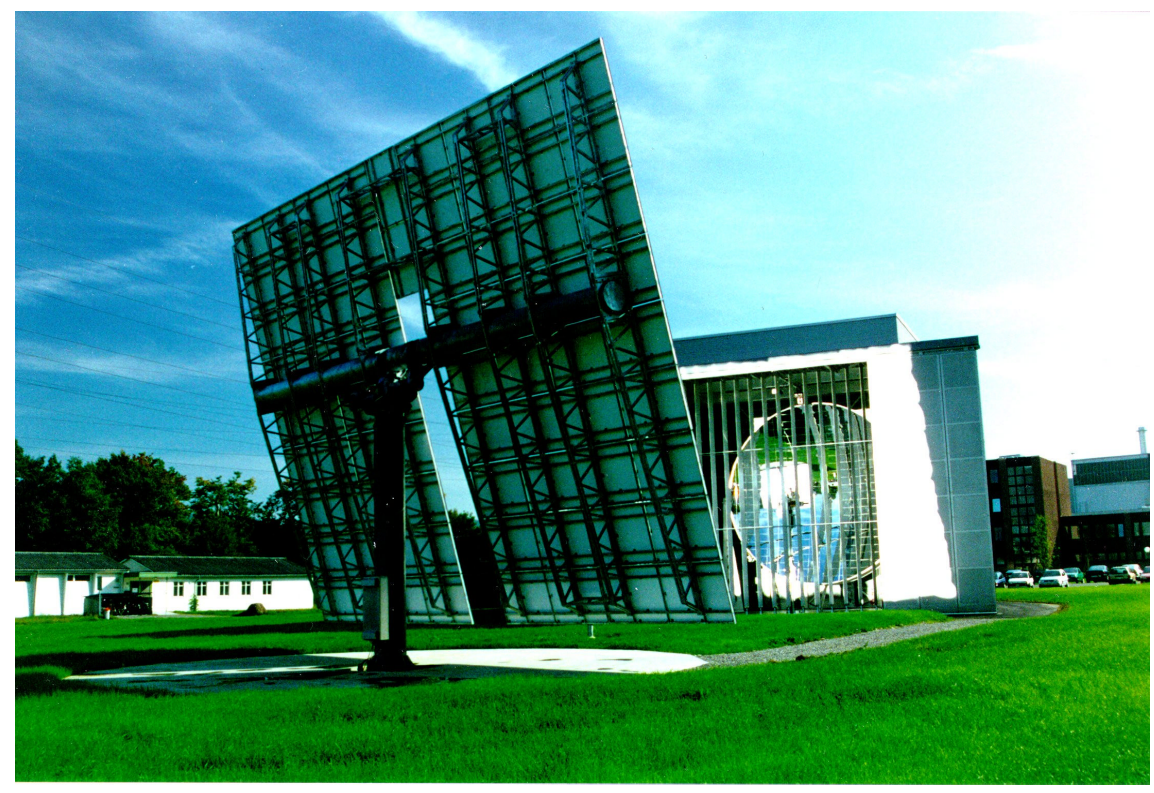

Abbildung 6: Der Hochfluss-Solarofen am Paul Scherrer Institut (PSI) in der Schweiz besteht aus einem Flachspiegel, welcher der Sonne nachgeführt wird, und einem stationären Parabolspiegel. Die solarthermische Leistung beträgt ca. $40 \mathrm{~kW}$ bei einer maximalen Konzentration von über 5,000 Sonnen. Die Solaranlagen am PSI werden für die experimentelle Untersuchung von Hochtemperatur-Prozessen in Solarreaktoren benutzt.

\section{"Veredelung" von fossilen Brennstoffen durch Sonnenenergie}

Die Ersetzung von fossilen Brennstoffen durch solare Brennstoffe, z. B. solaren Wasserstoff oder solare Metalle, ist ein längerfristiges Ziel, zu dessen Verwirklichung die Entwicklung neuartiger Technologien erforderlich ist. Es wird einige Zeit brauchen, bevor diese Methoden für kommerzielle Anwendungen reif sein werden. Aus strategischen Gründen ist es deshalb erstrebenswert, ein mittelfristiges Ziel zu formulieren, welches die Entwicklung von hybriden Prozessen beinhaltet. Als hybride Prozesse bezeichnen wir endotherme Prozesse, die fossile 
Brennstoffe ausschliesslich als chemische Reduktionsmittel verwenden, während die Solarenergie die benötigte Prozesswärme liefert. Durch die Zufuhr von Sonnenenergie wird somit die Qualität bzw. der Heizwert dieser Brennstoffe verbessert. Solche Brennstoffe sind deshalb sauberer als die ursprünglichen fossilen Brennstoffe.

Die Mischung von solaren und fossilen Energien schafft eine Verbindung zwischen den heutigen fossilen Technologien und den zukünftigen solarchemischen Technologien. Sie baut Brücken zwischen den heutigen und den zukünftigen Energiewirtschaften. Wir sind uns bewusst, dass bei den gegenwärtigen Preisen für fossile Energie die Brennstoffe aus konzentrierter Sonnenenergie nicht konkurrenzfähig sind. Solare Technologien werden jedoch schon bald eine ökonomisch sinnvolle Option darstellen, wenn die Kosten für fossile Energien auch die externen Kosten für die Verbrennung und den Abbau von fossilen Brennstoffen mit einbeziehen. Dies wird den Übergang von fossilen zu solaren Brennstoffen erleichtern und den Transfer der Solartechnologie an die Industrie beschleunigen. Abbildung 7 veranschaulicht unsere Forschungsstrategie, die einerseits das langfristige Ziel zur Herstellung von rein solaren Brennstoffen verfolgt, anderseits Wege sucht, um mittelfristig Erfolg versprechende Prozesse zu entwickeln, die sowohl fossile als auch solare Energie verwenden.

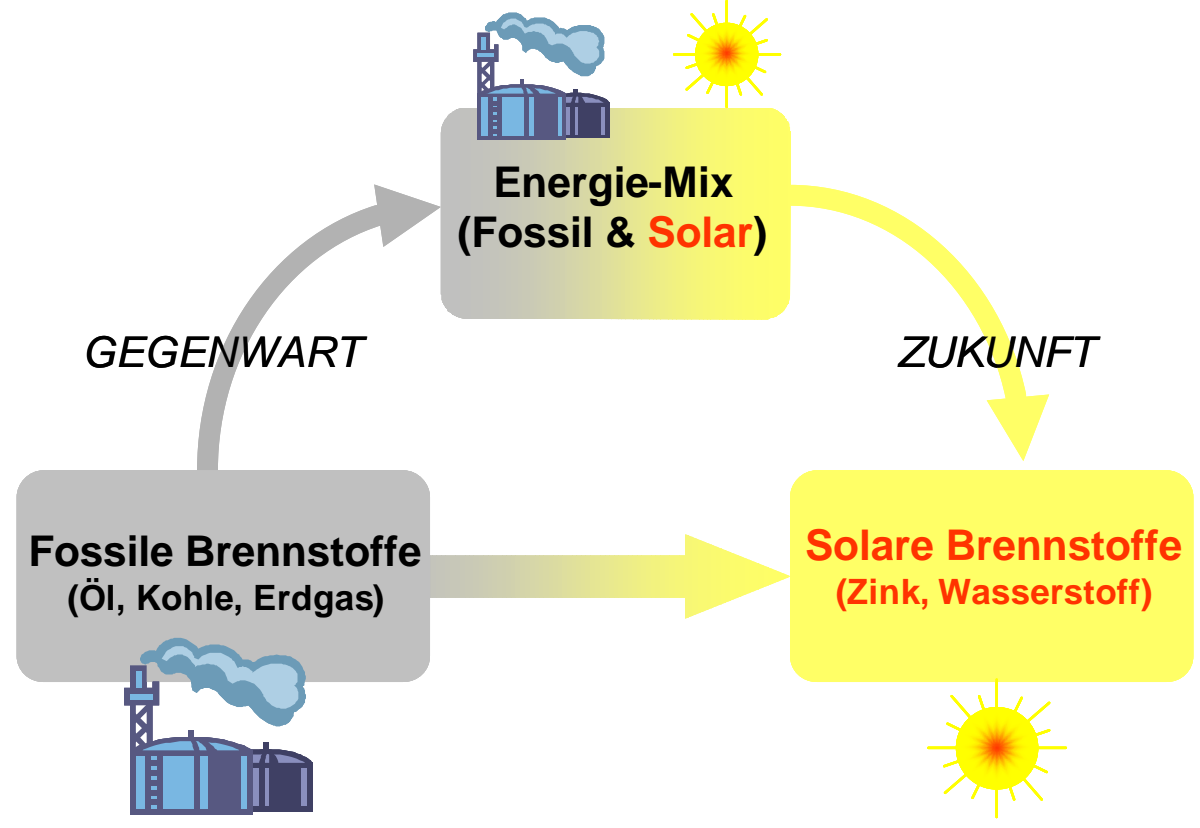

\begin{abstract}
Abbildung 7: Die Strategie zur Ersetzung von fossilen Brennstoffen durch solare Brennstoffe umfasst die Forschung auf zwei Pfaden: ein Pfad führt langfristig zu einer streng nachhaltigen Energieversorgung, während der andere Pfad mittelfristig eine Mischung von fossilen und solaren Energien zulässt.
\end{abstract}

Abbildung 8 zeigt einen Prozess, der eine Mischung von fossilen und solaren Energien enthält [6]. Man sieht, dass diesem Prozess nicht nur Sonnenlicht und Wasser, sondern auch Erdgas zugeführt wird. Allerdings wird das Erdgas nicht verbrannt, um Prozesswärme zu erzeugen; diese Arbeit leistet die Sonnenenergie. Statt dessen wird das Erdgas ausschliesslich dazu verwendet, um einerseits als Reduktionsmittel für das Zinkoxid und anderseits als Reservoir für synthetisches Gas (Synthesegas) zu dienen. Synthesegas besteht hauptsächlich aus Wasserstoff und Kohlenmonoxid und findet in der organischen Chemie breite Anwendung, so auch bei der Herstellung von Methanol, welches als vielversprechender Ersatz für Benzin als Fahrzeugtreibstoff gilt. Zink dient als wieder verwendbarer Brennstoff, der die Sonnenenergie, wie bereits erwähnt, speichert und leicht transportierbar ist. Die chemischen Produkte des 
Gesamtprozesses sind Methanol und Wasserstoff; sie werden mit Sonnenenergie energetisch verbessert und sind daher sauberer als die ursprünglichen fossilen Brennstoffe. Verglichen mit dem Heizwert von Erdgas zur Stromerzeugung, entlässt dieser solar unterstützte Prozess nur halb so viel $\mathrm{CO}_{2}$ in die Atmosphäre. Die Hauptreaktion dieses Prozesses geschieht im Solarreaktor, nämlich die kombinierte Reduktion des Zinkoxids und die Aufbereitung von Erdgas zur Herstellung von Zink und Synthesegas. Die Reaktion ist stark endotherm und läuft erst bei Temperaturen über $1250 \mathrm{~K}$ vollständig ab. Die technische Machbarkeit des Prozesses wurde mit einem $5 \mathrm{~kW}$ Solarreaktor nachgewiesen, der im Hochfluss-Solarofen des PSI getestet wurde (Abbildung 6). Die Resultate lassen den Schluss zu, dass die Reaktortechnologie für die Entwicklung im industriellen Massstab bereit ist [6].

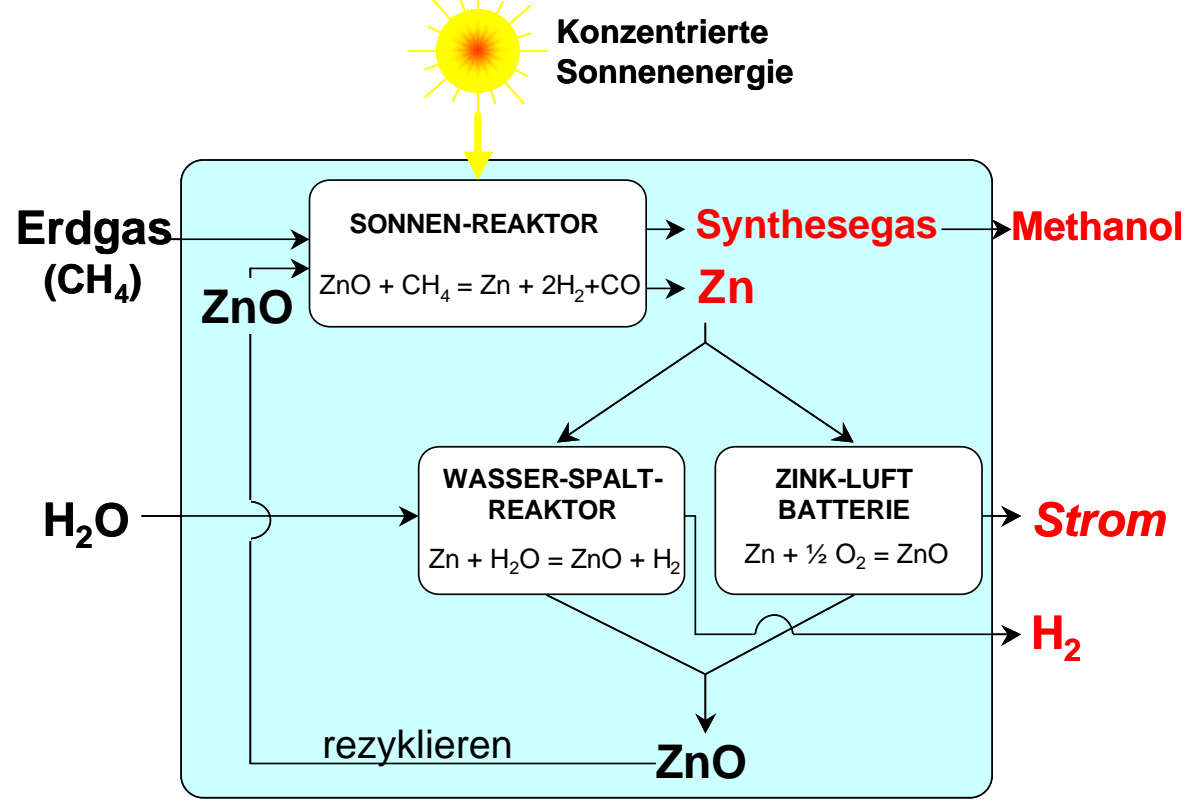

\begin{abstract}
Abbildung 8: Schema eines solaren Prozesses für die Herstellung von Zink, Synthesegas/Methanol und Wasserstoff/Strom. Erdgas und Wasser dienen als Rohmaterial, die Sonnenenergie als Quelle für die Hochtemperatur-Prozesswärme. Zwei Hauptschritte werden unterschieden: in einem ersten Schritt mit konzentrierter Sonnenenergie wird Zinkoxid mit Erdgas reduziert zu Zink und Synthesegas, wobei Synthesegas weiter zu Methanol verarbeitet wird; in einem zweiten Schritt wird mit dem Zink entweder Wasser gespalten und Wasserstoff produziert (der nicht mit Kohlenstoffoxiden verunreinigt ist), oder Zink wird in einer Zink/Luft Brennstoffzelle oder Batterie zur Erzeugung von elektrischer Energie verwendet. In beiden Fällen ist das Produkt Zinkoxid, welches in den solaren Schritt zurückgeführt wird. Mit diesem Prozess wird Sonnenenergie für die Speicherung und den Transport in solare Brennstoffe umgewandelt [7].
\end{abstract}

\title{
Solarthermische Wiederverwertung von Abfallstoffen
}

Feste Abfallstoffe aus vielfältigen Quellen (z. B. Verbrennungsrückstände von Hausmüll, entladene Batterien, schmutziger Schrott, kontaminierte Böden, Stäube und Schlämme, sowie andere Beiprodukte der metallurgischen Industrie) enthalten toxische Bestandteile, die nicht an die Umwelt abgegeben werden dürfen. Sie werden üblicherweise in Sondermülldeponien entsorgt, wo sie dauernd überwacht werden müssen. Allerdings haben begrenzter Lagerraum, steigende Lagerkosten sowie strengere Umweltvorschriften dazu geführt, dass nach Technologien geforscht wird, um toxische Abfälle wieder $\mathrm{zu}$ verwerten, statt sie für 
unbestimmte Zeit zu deponieren. Thermische Prozesse eignen sich gut für die Behandlung von komplexen Abfallstoffen. Abfälle mit kohlehaltigen Verbindungen lassen sich durch Pyrolyse und Vergasung in Synthesegas und Kohlenwasserstoffe umwandeln, die zu wertvollen synthetischen Chemikalien weiter verarbeitet werden können. Abfälle mit Metalloxiden können durch carbothermische Reduktion in Metalle, Nitride, Karbide und andere metallische Verbindungen umgewandelt werden. Die chemischen Produkte dieser Umwandlungen sind Ausgangsstoffe für eine Reihe von Fertigungsprozessen und können auch als Brennstoffe verwendet werden.

Geschlossene Stoffkreisläufe zur Wiederverwertung toxischer Abfallstoffe verlangen Prozesse, die bei hohen Temperaturen ablaufen. Die kommerziellen Verfahren in Hochöfen, Induktionsöfen, Lichtbogenöfen und Plasmaöfen verbrauchen enorme Mengen an Strom und Hochtemperatur-Prozesswärme und tragen demzufolge auch hauptsächlich zu den Emissionen von Treibhausgasen und anderen Schadstoffen bei. Im Gegensatz dazu liefert konzentrierte Solarstrahlung saubere Wärmeenergie bei den erforderlichen hohen Temperaturen, um diese komplexen Prozesse anzutreiben. In einem gemeinsamen Projekt entwickeln PSI, ETH und ein Industriepartner einen solarchemischen Reaktor, der die Wiederverwertung von Auto-Resh, entladenen Batterien und anderen toxischen Abfällen erlaubt. Besonders interessant ist Filterstaub, der beim Einschmelzen von Schrott in Lichtbogenöfen entsteht („Electric arc furnace dust", EAFD). Dieser Filterstaub ist eine Mischung aus Metalloxiden, die typisch etwa $50 \%$ toxische Metalle enthält, davon 10\% Blei und 38\% Zink [8]. Bei Experimenten im Solarofen des PSI ist es uns im April 2000 weltweit erstmals gelungen, mit konzentrierter Solarstrahlung im patentierten Solarreaktor Blei- und Zinkoxide aus EAFD bei 1500 bis $1600 \mathrm{~K}$ carbothermisch zu reduzieren (Abbildung 9). Als Produkt erhielten wir eine Mischung aus metallischem Zink und Blei, die als Energieträger oder Rohstoff weiterverwertet werden kann, sowie einen Rückstand mit einem Gehalt an toxischen Metallen von weniger als 4\%.

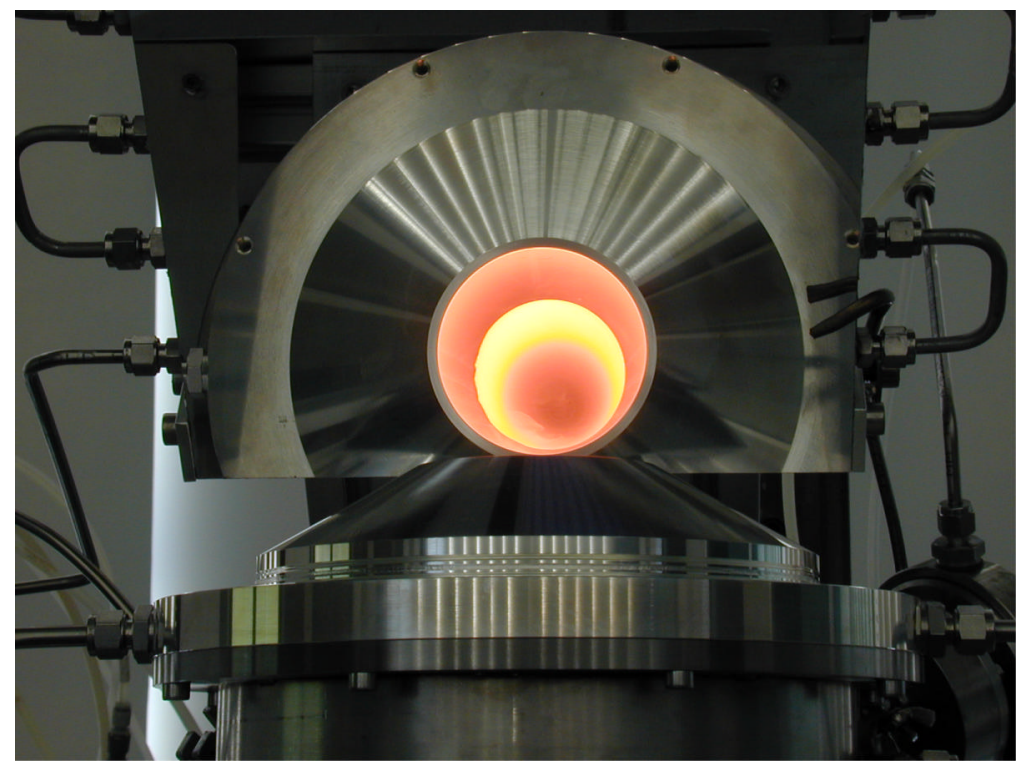

Abbildung 9: Solarreaktor im Solarofen des PSI, kurz nach Beendigung eines Experiments zur Wiederverwertung von Filterstaub aus Lichtbogenöfen (EAFD). Die konzentrierte Solarstrahlung heizt den Solarreaktor, der in der Brennebene des Parabolspiegels montiert ist, auf die erforderliche Temperatur von etwa $1500 \mathrm{~K}$. Die Reaktanden werden im Solarreaktor carbothermisch zu einer metallischen Zink-Blei-Mischung umgewandelt. Der Rückstand enthält weniger als $4 \%$ Schwermetalle. 
Die Zersetzung von Kalkstein (hauptsächlich $\mathrm{CaCO}_{3}$ ) ist der wichtigste endotherme Schritt bei der Herstellung von Kalk $(\mathrm{CaO})$ und Zement. Die Chemie des Kalzinierungsprozesses ist in Gl. (5) beschrieben:

$$
\mathrm{CaCO}_{3} \rightarrow \mathrm{CaO}+\mathrm{CO}_{2}
$$

Kürzlich haben PSI und ETH zusammen mit einem Industriepartner ein Projekt gestartet, um das Potential einer solaren Kalzinierungsanlage $\mathrm{zu}$ erkunden, bei der konzentrierte Sonnenenergie anstelle von kohlehaltigen Brennstoffen als Wärmequelle für die Herstellung von Kalk eingesetzt wird. Die Herausforderung besteht darin, eine kostengünstige Technologie zu entwickeln, die an eine bestimmte Gegend (z. B. in einem Entwicklungsland) angepasst und für einen bestimmten Zweck (z. B. die Herstellung von Kalk als Baumaterial) optimiert ist. Im Speziellen soll die wirtschaftliche Machbarkeit einer Kalzinierungsanlage mit 0.5 MW solarer Eingangsleistung untersucht werden. Zu diesem Zweck wird vorerst ein Solarreaktor im Leistungsbereich von 5 bis $10 \mathrm{~kW}$ entwickelt, der bei Temperaturen bis etwa $1600 \mathrm{~K}$ betrieben werden soll. Die experimentellen Resultate der Versuche im Solarofen des PSI werden dann mit einem numerischen Modell kombiniert. Zusammen mit aktueller Information über die zu erwartenden Kosten der solaren Technologie lassen sich dann die Gestehungskosten einer solaren Kalzinierungsanlage im Leistungsbereich von 0.5 MW abschätzen. Bis zum Jahr 2003 soll entschieden werden, ob eine Pilotanlage zur solaren Herstellung von Kalk errichtet werden soll.

\section{SCHLUSSFOLGERUNG}

Konzentrierte Solarstrahlung kann die Prozesswärme für energieaufwändige chemische Prozesse liefern, die bei hohen Temperaturen ablaufen. Dadurch lässt sich der Ausstoss von Treibhausgasen und anderen Schadstoffen vermeiden, der durch die Verbrennung von fossilen Brennstoffen zur Wärme- und Stromproduktion verursacht wird. Mittels thermochemischer Prozesse lässt sich Sonnenenergie in speicherbare und transportierbare chemische Brennstoffe umwandeln. Es bestehen gute Aussichten, dass die solaren thermochemischen Technologien zur Herstellung von chemischen Brennstoffen gegenüber anderen, konventionellen Herstellungsverfahren konkurrenzfähig werden. Voraussetzung dazu ist allerdings, dass die Kosten für fossile Energieträger sowohl die externen Kosten für die Verbrennung und den Abbau von fossilen Brennstoffen mit einbeziehen, als auch die Kosten für die Vermeidung von $\mathrm{CO}_{2}$-Emissionen und für die Verminderung von Schadstoffen berücksichtigen.

Unsere Forschung auf dem Gebiet der Hochtemperatur-Solarchemie versucht einerseits, die Verbindung zwischen bestehender und zukünftiger Technologie durch die Mischung von fossilen Brennstoffen und Sonnenlicht herzustellen, anderseits entwickelt sie das wissenschaftliche und technologische Know-how für ein radikal neues Rezept, um aus Sonnenlicht und Wasser solare Brennstoffe zu produzieren. Beide Konzepte haben das gleiche Ziel: die Entwicklung von Brennstoffen, die mit einer sauberen, universellen und nachhaltigen Energiequelle hergestellt werden können.

Dank - Die Forschungsprogramme auf dem Gebiet der Solarchemie am PSI und an der ETH werden teilweise finanziert vom BFE - Schweizer Bundesamt für Energie, vom BBW - Schweizer Bundesamt für Bildung und Wissenschaft, von der Baugarten Stiftung, und von industriellen Partnern. 


\section{REFERENZEN}

[1] Steinfeld A., Palumbo R.: Fuels from Sunlight and Water. In: Sun at Work in Europe (1997), Vol.12, No.2, S.8-10

[2] Steinfeld A., Palumbo R.: Solar Thermochemical Process Technology. In: Encyclopaedia of Physical Science \& Technology, R. A. Meyers Ed., Academic Press, ISBN 0-12227410-5, Vol. 15, pp. 237-256, 2001.

[3] Steinfeld A., Kuhn P., Reller A., Palumbo R., Murray J., Tamaura Y.: Solar-processed metals as clean energy carriers and water-splitters. In: Int. J. Hydrogen Energy (1998), Vol.23, No.9, S.767-774

[4] Palumbo, R., Lédé, J., Boutin, O., Elorza-Ricart, E., Steinfeld, A., Möller, S., Weidenkaff, A., Fletcher, E.A., and Bielicki, J.: The production of Zn from ZnO in a high-temperature solar decomposition quench process - I. The scientific framework for the process. In: Chemical Engineering Science (1998), Vol.53, No.14, S.2503-2517

[5] Haueter, P., Moeller, S., Palumbo, R., and Steinfeld, A.: The production of zinc by thermal dissociation of zinc oxide - Solar chemical reactor design. In: Solar Energy (1999), Vol.67, Nos.1-3, S.161-167

[6] Steinfeld A., Brack M., Meier A., Weidenkaff A., Wuillemin D.: A solar chemical reactor for co-production of zinc and synthesis gas. In: Energy - The International Journal (1998), Vol.23, S.803-814

[7] Steinfeld A., Frei A., Kuhn P., and Wuillemin D.: Solar thermal production of zinc and syngas via combined $\mathrm{ZnO}$-reduction and $\mathrm{CH}_{4}$-reforming processes. In: Int. J. Hydrogen Energy (1995), Vol.20, No.10, S.793-804

[8] Schaffner B., Hoffelner W., Sun H., Steinfeld A.: Recycling of Hazardous Solid Waste Material Using High-Temperature Solar Process Heat. 1. Thermodynamic Analysis. In: Environ. Sci. Technol. (2000), Vol.34, S.4177-4184 